\title{
Hyperbilirubinemia as a Significant Indicator in Diagnosis of Complicated Acute Appendicitis: A Prospective Study
}

\author{
Fady Makram, MD, FACS; Ahmed Shoka, MD, MRCS; Mohamed Matar, MD, FACS \\ Department of General Surgery, Ain Shams University, Cairo, Egypt
}

Background: Acute appendicitis is the most common cause of acute abdomen in young adults. In recent years, studies emerged showing that elevated serum total bilirubin levels could indicate a complicated case of acute appendicitis. An elevated serum total bilirubin that is not explained by liver disease or biliary obstruction can be present in many patients of acute appendicitis.

Patients and methods: This is a prospective interventional non-randomized non-controlled study conducted on 175 consecutive cases of acute appendicitis admitted to the emergency unit in the Department of General Surgery at Ain Shams University hospitals, Cairo, Egypt in the period from July 2014 to August 2016. Liver function tests were done as a part of the preoperative investigations. Clinical diagnosis was confirmed postoperatively by histopathological examination. Their clinical and investigative data were analyzed, The level of significance was set at $\mathrm{P}<0.05$.

Results: Serum total bilirubin was found to be significantly elevated in cases of complicated acute appendicitis.

Conclusion: Serum total bilirubin is a new important adjunct in the diagnosis of complicated acute appendicitis.

Key words: Hyperbilirubinemia, acute appendicitis, acute abdomen.

\section{Introduction}

Appendectomy is one of the most commonly performed abdominal surgeries in the emergency settings, delay in diagnosis and surgery for this condition may lead to various complications like perforation, intraabdominal abscesses, small bowel obstruction and peritonitis causing an increase in morbidity and even mortality of these patients. ${ }^{1}$

On the other hand, too aggressive surgical approach may lead to an increased rate of negative appendectomies. The incidence of perforated acute appendicitis in adults has been reported from $13-37 \%$ or higher. $^{2}$

Hyperbilirubinemia is the result of imbalance between production and excretion of bilirubin by the liver. Elevated serum bilirubin level and jaundice are commonly observed in patients suffering from a septic condition. ${ }^{3}$

Acute appendicitis is the most common intraabdominal infectious focus in a surgical patient, Escherichia coli and Bacteroides fragilis are the most frequent bacterial isolates in this condition. ${ }^{4}$ Bacteraemia is known to cause endotoxemia leading to impaired excretion of bilirubin from the bile canaliculi. ${ }^{5}$

Few case reports have described hyperbilirubinemia in patients with severe postoperative infection after appendectomy or with complicated acute appendicitis.

The aim of this study was to establish the role of serum total bilirubin level as an additive diagnostic tool to diagnose complicated acute appendicitis and to accelerate early intervention, the establishment of a possible role of hyperbilirubinemia as a predictor of complicated acute appendicitis has been stressed so that level of serum total bilirubin on admission can be used in combination with leucocytic count, pelviabdominal ultrasonography and computed tomography to help determine the presence of complication and to aid suitable management.

\section{Patients and methods}

This prospective interventional non-randomized non-controlled study was conducted in the Emergency Unit in the Department of General Surgery at Ain Shams University hospitals, Cairo, Egypt in the period from July 2014 to August 2016. All patients were admitted with the diagnosis of acute appendicitis. Serum total bilirubin was done in addition to the routine investigations including total leukocytic count and pelviabdominal ultrasonography. 
Patients with elevated serum total bilirubin were investigated by hepatitis viral markers. After the operation, all specimens were sent for histopathological examination and only cases with acute appendicitis or complicated acute appendicitis were included in our study.

Exclusion criteria: All patients documented to have a history of jaundice or liver disease, chronic alcoholism, hemolytic disease, acquired or congenital biliary disease, viral hepatitis, and patients with cholelithiasis.

Also, patients with normal appendix which was proved by postoperative histopathological examination were excluded from the study.

Statistical methodology: Data were coded and entered using the statistical package SPSS (Statistical Package for the Social Science) version 23. Data was summarized using mean, minimum and maximum in quantitative data and using frequency (count) and relative frequency (percentage) for categorical data.

\section{Results}

Patients' demographics: (Table 1)

Our study consisted of 175 patients: 84 males (48\%) and 91 females (52\%) with acute appendicitis that was proved postoperatively by histopathological examination.

The age varied between 14 years and 62 years with mean age 28.3 years.

Table 1: Patients' demographics

\begin{tabular}{cccc}
\hline & & Number & Percentage \\
\hline \multirow{3}{*}{ Sex } & Male & 84 & $48 \%$ \\
& Female & 91 & $52 \%$ \\
\multirow{2}{*}{ Age } & Mean & Minimum & Maximum \\
& 84 & 14.00 & 62.00 \\
\hline
\end{tabular}

Duration of symptoms ranged from 6 hours to maximum 7 days. Among the 175 cases, according to the intraoperative findings and the postoperative histopathological results, 115 cases $(65.71 \%)$ were non-complicated acute appendicitis while 60 cases (34.28\%) were complicated either by abscess formation or appendicular perforation.

Among the 60 complicated cases, hyperbilirubinemia was found in 51 patients (sensitivity $=85 \%$ ), while normal bilirubin was found in 9 patients $(15 \%)$, on the other hand, out of the 115 non-complicated cases, normal bilirubin was found in 98 patients (specificity $=85.21 \%$ ) while it was elevated in 17 patients $(14.78 \%)$ with accuracy $85.14 \%$.

The mean total bilirubin for patients with non- complicated acute appendicitis was 0.77 and that for complicated cases was $1.62(p=0.001)$ which was a significant rise in serum total bilirubin in patients with complicated acute appendicitis (Table 2).

Table 2: Serum total bilirubin in complicated and non-complicated cases

\begin{tabular}{|c|c|c|c|}
\hline & Mean & SD & P-value \\
\hline $\begin{array}{l}\text { Complicated acute } \\
\text { appendicitis }\end{array}$ & 1.62 & 0.27 & 077 \\
\hline $\begin{array}{l}\text { Non-complicated } \\
\text { acute appendicitisv }\end{array}$ & 0.77 & 0.15 & 0.77 \\
\hline
\end{tabular}

\section{Discussion}

The incidence of acute appendicitis differs, but it is highly prevalent, occurring in 7 to 22 out of 10,000 people. ${ }^{6}$

Non-complicated acute appendicitis can be treated by an appendectomy without a long recovery period, whereas, complicated appendicitis can cause different complications that can lead to lifethreatening conditions. ${ }^{7}$

Recent developments in the diagnosis of acute appendicitis with the assistance of different radiological tools such as ultrasonography and computed tomography have reduced the rate of negative appendectomies. Although the known diagnostic accuracy of ultrasonography varies depending on the patient population studied, a meta-analysis reported an overall ultrasonographic specificity of $92 \%$ and a sensitivity of $85 \%{ }^{8}$

Because of the development of helical CT, the accuracy and the effectiveness of diagnosing appendicitis have already defeat the limitation of ultrasonography, with sensitivities of 90 to $99 \%$ and specificities of 91 to $99 \% .^{9}$

However, a recent study by Pritchett et al., 2010 10 reported that the widespread use of computed tomography scanning in acute appendicitis increases the care cost and the hospital stay and delays the time to surgical intervention. Because clinical examinations and laboratory investigations are still acknowledged as being of the utmost importance in the diagnostic process, ${ }^{11}$ we tried to find key laboratory tests that would allow us to anticipate the severity of acute appendicitis.

Miller and Irvine, $1969^{12}$ first reported that jaundice was common in patients with severe appendicitis, Recently Sand et al., $2009^{13}$ reported a relatively high incidence of hyperbilirubinemia $(24.9 \%)$ from an analysis of 538 acute appendicitis patients of whom $50.7 \%$ were verified as having perforated appendicitis. 
He derived hypothesis that elevated serum total bilirubin levels can be associated with appendicular perforation. They explained the raised serum bilirubin levels by the invasion of gram negative bacteria through muscularis propria of the appendix leading to the direct invasion or translocation of germs through portal system and liver interfering with bilirubin excretion through bile ducts by endotoxin action.

Recently, few studies have investigated the role of serum total bilirubin in diagnosis of perforated appendicitis, one study has found hyperbilirubinemia to be an important predictive marker for perforated and necrotizing appendicitis. In this series of 157 patients, the odds of gangrenous and perforated appendicitis were found to be 3 times higher for patients having hyperbilirubinemia than those with normal bilirubin level. ${ }^{14}$

A retrospective Cohort study performed in two centers involving 155 cases of perforated appendicitis and 570 cases of non-perforated appendicitis compared hyperbilirubinemia, C-reactive protein, leucocytic count and age as a marker of perforated appendicitis, they concluded that hyperbilirubinemia is a statistically significant marker of perforated appendicitis, but they also found that C-reactive protein to be a superior marker of perforation than hyperbilirubinemia. ${ }^{15}$

Emmanuel A et al., $2011^{16}$ concluded that the positive predictive value of hyperbilirubinemia in appendicular perforation was found to be $91 \%$ in his study.

Multivariate analysis done by Young Ran Hong et al., $2012^{17}$ stated that the Systemic Inflammatory Response Syndrome (SIRS) score and hyperbilirubinemia are significant predictors of appendicular perforation, whereas the leucocytic count and segmented neutrophils, which are used as markers of acute inflammation frequently, were not statistically significant. However, as the SIRS score itself is a sum of four figures with four criteria, using it as an independent variable would be inappropriate. They confirmed that hyperbilirubinemia has a predictive value in acute appendicitis patients.

Diagnostic meta-analysis of 4974 patients was done by Salvatore G et al., 2013, ${ }^{18}$ eight studies were used for this pooled analysis: seven of them were retrospective and only one prospective cohort with clear heterogeneity in settings and methods (e.g. country, time since diagnosis, operative indications). They concluded that alone, hyperbilirubinemia is not a strong enough predictor, but it might be useful when combined in a scoring system, so, it is considered that further studies would be useful especially when there are poor resource settings with limited availability of other imaging studies.

\section{Conclusion}

Serum total bilirubin should be routinely performed as a part of the preoperative assessment in cases of suspected acute appendicitis. It can serve as an important marker of complicated acute appendicitis.

\section{References}

1. Korner $\mathrm{H}$, Sondenaa $\mathrm{K}$, Soreide JA, Andersen E, Nysted $A$, Lende $T H$, et al: Incidence of acute non-perforated and perforated appendicitis: Age-specific and sex-specific analysis. World $\boldsymbol{J}$ Surg 1997; 21: 313-317.

2. Temple $\mathrm{CL}$, Huchcroft $\mathrm{SA}$, Temple WJ: The natural history of appendicitis in adults - A prospective study. Ann Surg 1995; 221: 278281.

3. Whitehead MW, Hainsworth I, Kingham JG: The causes of obvious jaundice in South West Wales: Perceptions versus reality. Gut 2001; 48: 409-413.

4. Cheng CW, Lin HS, Ye JJ, Yang CC, Chiang PC, Wu TS, et al: Clinical significance of and outcomes for Bacteroides fragilis bacteremia. J Microbiol Immunol Infect 2009; 42: 243-250.

5. Franson TR, Hierholzer WJ, LaBrecque DR: Frequency and characteristics of hyperbilirubinemia associated with bacteremia. Rev Infect Dis 1985; 7: 1-9.

6. Buckius MT, McGrath B, Monk J, Grim R, Bell $T$, Ahuja V: Changing epidemiology of acute appendicitis in the United States: study period 1993-2008. J Surg Res 2012; 175: 185-190.

7. Fike $F B$, Mortellaro $V E$, Juang $D$, Sharp SW, Ostlie DJ, St Peter SD: The impact of postoperative abscess formation in perforated appendicitis. J Surg Res 2011; 170: 24-26.

8. Orr RK, Porter D, Hartman D: Ultrasonography to evaluate adults for appendicitis: Decision making based on meta-analysis and probabilistic reasoning. Acad Emerg Med 1995; 2: 644-650.

9. Lane MJ, Liu DM, Huynh MD, Jeffrey RB Jr, Mindelzun RE, Katz DS: Suspected acute appendicitis: Nonenhanced helical CT in 300 consecutive patients. Radiology 1999; 213: 341-346. 
10. Pritchett CV, Levinsky NC, Ha YP, Dembe AE, Steinberg SM: Management of acute appendicitis: The impact of CT scanning on the bottom line. J Am Coll Surg 2010; 210: 699-705.

11. Kalliakmanis V, Pikoulis E, Karavokyros IG, Felekouras E, Morfaki P, Haralambopoulou G, et al: Acute appendicitis: The reliability of diagnosis by clinical assessment alone. Scand J Surg 2005; 94: 201-206.

12. Miller DF, Irvine RW: Jaundice in acute appendicitis. Lancet 1969; 1: 321-323.

13. Sand M, Bechara GF, Holland-Letz $T$, Sand D, Mehnert G, Mann B: Diagnostic value of hyperbilirubinemia as a predictive factor for appendiceal perforation in acute appendicitis. Am J Surg 2009; 198: 193-198.

14. Käser SA, Fankhauser G, Willi N, Maurer CA: Creactive protein is superior to bilirubin for anticipation of perforation in acute appendicitis.
Scand J Gastroenterol 2010; 45: 885-892.

15. Khan S: Elevated serum bilirubin in acute appendicitis: a new diagnostic tool. Kathmandu Univ Med J 2008; 6: 161-165.

16. Emmanuel A, Murchan P, Wilson I, Balfe P: The value of hyperbilirubinemia in the diagnosis of acute appendicitis. Ann R Coll Surg Engl 2011; 93: 213-217.

17. Hong YR, Chung CW, Kim JW, Kwon C, Ahn DO, Kwon SW, et al: Hyperbilirubinemia is a significant indicator for the severity of acute appendicitis. J Korean Soc Coloproctol 2012; 28: 247-252.

18. Giordano S, Pääkkönen $M$, Salminen $P$, Grönroos JM: Elevated serum bilirubin in assessing the likelihood of perforation in acute appendicitis: A diagnostic meta-analysis. International Journal of Surgery 2013; 11: 795-800. 